\title{
EFECTO DE UN PROGRAMA DE ACTIVIDAD FÍSICA DE ALTA INTENSIDAD EN LA ATENCIÓN SELECTIVA DE JÓVENES CON TDAH
}

\author{
Sara Suárez-Manzano \\ Facultad de Humanidades y Ciencias de la Educación. \\ Dpt. Didáctica de la Expresión M. P. y Corporal \\ Universidad de Jaén \\ ssuarez@ujaen.es
}

Fecha de Recepción: 1 Febrero 2018

Fecha de Admisión: 10 Abril 2018

\section{RESUMEN}

El objetivo de este estudio fue analizar el efecto a corto plazo de 16 minutos de "high-intensity interval training" (HIIT) en la atención selectiva de niños diagnosticados con Trastorno por Déficit de Atención e Hiperactividad (TDAH). Este estudio tiene diseño experimental randomizado. La muestra estaba formada por 20 niños diagnosticados TDAH (6-12 años). El grupo control $(n=10)$ vio un video documental y el grupo experimental $(n=10)$ hizo 16 minutos HIIT con monitor cardiaco (80$95 \%$ frecuencia cardiaca máxima). La atención selectiva se midió utilizando el test d2. El análisis de medidas repetidas ANOVA 2 veces (pre-test vs. post-test) $\times 2$ grupos (grupo control vs. grupo experimental). Los resultados han mostrado que practicar 16 minutos de HIIT tiene un efecto de mejora inmediato en la atención, disminuyendo el número de omisiones y errores y aumentando el número de aciertos. Se concluye, que realizar 16 minutos de HIIT mejora la atención selectiva inmediata en niños escolares diagnosticados TDAH. Se recomienda promover en el contexto educativo la práctica de ejercicio físico, en concreto, realizar 16 minutos de HIIT al comienzo de la jornada escolar. Este ejercicio de corta duración y que no requiere grandes recursos materiales ni humanos podría ayudar a mejorar la atención y motivación de niños y niñas en clase.

Palabras clave: ejercicio físico; atención selectiva; estudiantes; colegio; hiperactividad

\section{ABSTRACT}

Effect of a program of Physical Activity of high intensity in the selective attention of young people with ADHD.

The aim of this study was to analyse the short-term effect of 16 minutes of high-intensity interval training (HIIT) in the selective care of children diagnosed with Attention Deficit Hyperactivity Disorder (ADHD). This study has a randomized experimental design. The sample consisted of 20 
children diagnosed with ADHD (6-12 years). The control group $(n=10)$ watched a documentary video and the experimental group $(n=10)$ did 16 minutes HIIT with cardiac monitor $(80-95 \%$ maximum heart rate). Selective attention was measured using the $\mathrm{d} 2$ test. The analysis of repeated measures ANOVA 2 times (pre-test vs. post-test) $\times 2$ groups (control group vs. experimental group). The results have shown that practicing 16 minutes of HIIT has an effect of immediate improvement in attention, decreasing the number of omissions and errors and increasing the number of correct answers. It is concluded that performing 16 minutes of HIIT improves immediate selective attention in school children diagnosed with ADHD. It is recommended to promote the practice of physical exercise in the educational context, specifically, to perform 16 minutes of HIIT at the beginning of the school day. This exercise of short duration and that does not require great material or human resources could help to improve the attention and motivation of children in class.

Keywords: physical exercise; selective attention; students; school; hyperactivity

\section{INTRODUCCIÓN}

El Trastorno por Déficit de Atención e Hiperactividad (TDAH) es un trastorno del neurodesarroIlo que afecta aproximadamente al 5\% de la población infantil (6 - 12 años) (Polanczyk, de Lima, Horta, Biederman, y Rohde, 2007). La prevalencia es mayor en niños que en niñas, concretamente, por cada niña diagnosticada son diagnosticados tres niños (Wittchen et al., 2011). Este trastorno está caracterizado por presentar un patrón persistente de inatención y/o hiperactividad-impulsividad que interfiere con el funcionamiento o desarrollo de las capacidades sociales y académicas / laborales (DSM5, 2013).

Se asocia por tanto a bajos niveles de atención en los diferentes ámbitos diarios del niño, en la escuela, en el hogar y en reunión con sus iguales (DSM5, 2013). En el ámbito escolar, en particular en la etapa preescolar _entre los tres y seis años_, estos niños manifiestan comportamiento disruptivo, berrinches, continua búsqueda de atención por parte de adultos y compañeros, el incumplimiento reiterativo de normas y tareas. Además, la hiperactividad e hipercinesia que manifiestan estos niños suele asociarse con rechazo por parte de los compañeros y un bajo rendimiento académico desde la escuela primaria (Egger y Angold, 2006).

Durante los últimos años, ha surgido una nueva corriente que aboga por la Actividad Física (AF) como tratamiento complementario/sustitutivo de la farmacología. Esta corriente se basa en los beneficios que aporta la realización de AF y especialmente a intensidad de moderada a vigorosa _ frecuencia cardiaca > 75\% de la frecuencia cardiaca máxima _ os niños diagnosticados TDAH presentan a nivel cerebral _ corteza prefrontal y lóbulo central estriado__ niveles de dopamina y noradrenalina más bajos que los niños sin dificultades de aprendizaje, lo que sumado a un estado de inquietud y ansiedad dificulta las tareas que requieren atención (Woleaich et al., 2005). Partiendo de que la práctica de AF aumenta los niveles de dopamina y noradrenalina (Woleaich et al., 2005) han surgido diversos estudios muestran esta relación positiva entre fitness y atención selectiva (Vanhelst et al., 2016). Piepmeier et al. (2015) comprobaron en un grupo de 32 adolescentes (10.75 \pm 2.27 años) diagnosticados TDAH, que la realización de ejercicio aeróbico _ 30 minutos de pedaleo en cicloergometro, manteniendo aproximadamente la intensidad al $68 \%$ de la frecuencia cardiaca máxima teórica calculada con la fórmula de Karvonen, Kentala y Mustala (1957), monitorizados con pulsómetro POLAR observaron en el grupo experimental mejoras en atención Stroop Test y Tower of London.

Sin embargo, al menos que nos conste a nosotros, no existe un trabajo que analice el efecto inmediato que tiene la realización de 16 minutos de AF de carácter interválico de alta intensidad, utilizando ejercicios de autocarga de forma aeróbica sobre los niveles de concentración en niños diag- 
nosticados TDAH. La mayoría de los trabajos se centras en actividades de más larga duración y de intensidad moderada, como el pedalear en cicloergometro (Piepmeier et al., 2015) o caminar / trotar en tapiz rodante (Pontifex et al., 2013).

Por tanto, el objetivo de este trabajo de investigación fue responder a la siguiente pregunta "¿Un programa de Actividad Física interválica de alta intensidad mejora la atención selectiva de niños escolares diagnosticados TDAH?". Este trabajo se centra en niños varones porque la prevalencia es mayor en chicos que en chicas. La franja de edad escogida ha sido la etapa escolar comprendida entre los seis y doce años porque en la etapa primaria, a partir de los seis años, aumentan los conflictos escolares y la dependencia, factores que se asocian con malos resultados académicos y problemas conductuales que seguirán aumentando en los años siguientes (Hamre y Pianta, 2001). Conocer el efecto de realizar AF interválica de alta intensidad en niños diagnosticados TDAH podría ayudar a planificar los horarios de tareas de estos estudiantes, de tal modo que pudieran aprovechar los beneficios del ejercicio físico para realizar las tareas que requieran mayores niveles de atención.

\section{METODOLOGíA}

\section{Diseño}

Este estudio empleó un diseño experimental con intervención. Hubo dos grupos a los que los participantes fueron asignados de forma aleatoria. El Grupo Control $(n=10)$ visualizó un video documental de 20 minutos de duración, y el Grupo de Experimental $(n=10)$ hizo un calentamiento de cuatro minutos, seguido de 16 minutos de High Intensity Interval Training (HIIT) o lo que es lo mismo, AF interválica de alta intensidad. Este estudio fue aprobado por el Comité de Bioética de la Universidad de Jaén. El diseño cumple con la normativa española de investigación clínica en humanos (Ley 14/2007, 3 de julio, Investigación Biomédica), con las normas de protección de datos privados (Ley Orgánica 15/1999) y con los principios de la Declaración de Helsinki (Versión 2013, Brasil). La distribución de la intervención se puede ver en la figura 1.

Tabla 1.

Características descriptivas de la muestra. Los valores se expresan como media \pm desviación estándar.

\begin{tabular}{|c|c|c|c|c|c|c|c|c|c|}
\hline \multirow[b]{2}{*}{ Edad (años) } & \multicolumn{3}{|c|}{$\begin{array}{c}\text { Total } \\
(n=30)\end{array}$} & \multicolumn{3}{|c|}{$\begin{array}{c}\text { Control } \\
(n=10)\end{array}$} & \multicolumn{3}{|c|}{$\begin{array}{c}\text { Experimental } \\
(n=10)\end{array}$} \\
\hline & 10.15 & \pm & 1.18 & 10.20 & \pm & 1.23 & 10.10 & \pm & 1.20 \\
\hline Peso (kg) & 34.81 & \pm & 5.54 & 35.47 & \pm & 6.20 & 34.15 & \pm & 5.04 \\
\hline
\end{tabular}


Figura 1. Flow de participación en el estudio

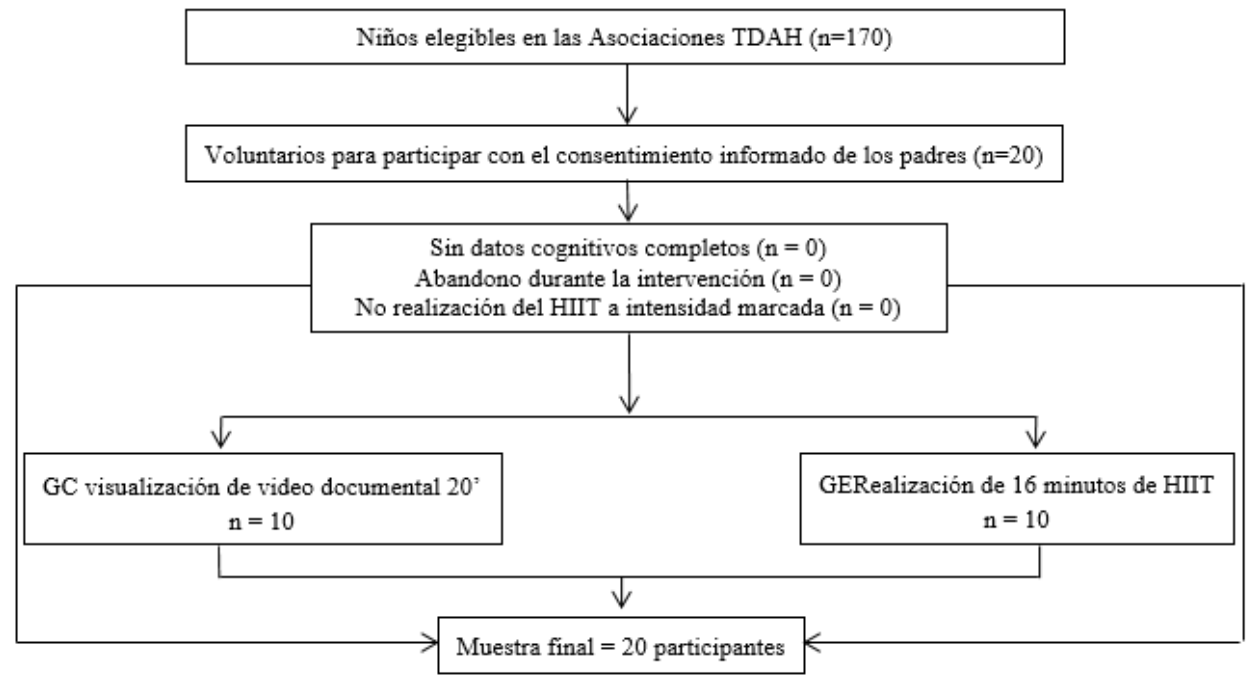

\section{Participantes}

Una muestra de 20 jóvenes diagnosticados TDAH por especialistas acreditados siguiendo las directrices de $D S M-5 \circledR$. Las características antropométricas y sociodemográficas de los participantes se muestran en la Tabla 1. Todos los niños que participaron en el estudio completaron todas las mediciones ( $n=20$ ), con una edad media de $10.15 \pm 1.18$ años (rango $9-12$ años), y un IMC de $18.68 \pm 6.62 \mathrm{~kg} / \mathrm{m}^{2}$ (Must y Anderson, 2006). Los escolares tienen un peso medio de $34.81 \pm 5.54$ $\mathrm{kg}$ y una talla media de $1.36 \pm 0.07 \mathrm{~m}$.

Tabla 1.

Características descriptivas de la muestra. Los valores se expresan como media \pm desviación estándar.

\begin{tabular}{|c|c|c|c|c|c|c|c|c|}
\hline Talla (m) & 1.36 & + & .07 & 1.37 & \pm & .09 & 1.36 & $\pm \quad .07$ \\
\hline $\operatorname{IMC}\left(\mathrm{kg} / \mathrm{m}^{2}\right)$ & 18.68 & \pm & 2.62 & 18.97 & \pm & 2.68 & 18.40 & 2.67 \\
\hline
\end{tabular}

Nota: IMC $=$ Índice de Masa Corporal

\section{Medidas}

EI IMC se calculó con el peso y la altura [peso $(\mathrm{kg}) /$ altura $\left(\mathrm{m}^{2}\right)$ ]. Se utilizó un aparato de pesaje ASIMED® B-tipo-clase III (España) y un medidor de altura portátil SECA 214 (SECA® Ltd, Alemania), respectivamente. A los participantes se les realizaron ambas mediciones descalzos y vestidos con ropa deportiva ligera.

\section{Atención}

Para evaluar la atención selectiva se utilizó Brickenkamp $s$ d2 test en versión española (Seisdedos, 2012). Este test se llevó a cabo utilizando una plantilla que consta de 14 líneas de 47 letras cada una y un total de 658 letras separadas todas a una misma distancia. Las letras que apa- 
recen son " $p$ " $y$ " $d$ ", acompañadas de una o dos comillas en la zona superior y/o inferior. Cada participante tenía que señalar todas aquellas letras "d" que tengan dos comillas arriba, dos comillas abajo, 0 una comilla arriba y otra abajo. La duración total del test es de cuatro minutos y 20 segundos. Las variables obtenidas fueron el número total de aciertos (TA), el número total de errores por Omisiones $(0)$ _ símbolos que debería de haber señalado, pero no señalaron_ y el número total de errores por Comisiones (C) _ símbolo que no debían de señalar, pero sí señalaron _ . La fiabilidad test-retest $(48 \mathrm{~h}, \mathrm{n}=21)$ fue 0.922 .

\section{Condición HIIT}

La condición de entrenamiento realizada por el Grupo Experimental fue a través del método HIIT. La sesión comenzó con una breve actividad de calentamiento de 4 minutos, incluyendo desplazamientos, ejercicios de trote / carrera y estiramientos dinámicos a intensidad media. La parte principal de la sesión fue de 16 minutos de duración de HIIT, de tal modo que se realizaron cuatro series de cada ejercicio propuesto. La relación trabajo-descanso fue de 30:30 segundos, es decir, 30 segundos de trabajo seguido de 30 segundos de descanso (Costigan et al., 2016). Este trabajo de HIIT incluyó una combinación de ejercicios de entrenamiento cardiorrespiratorio, motor y coordinativo, porque son los componentes de acondicionamiento físico que mayormente mejoran las funciones ejecutivas en niños diagnosticados TDAH (Suarez-Manzano et al., 2018). El ejercicio se repitió tantas veces como fue posible durante los 30 segundos de trabajo. La sesión de HIIT fue impartida por dos licenciados en ciencias del deporte especializados en este método de investigación y con experiencia superior a 2 años de trabajo con niños. Los participantes llevaban colocados monitores de frecuencia cardíaca Seego Realtracksystems $®$ (España) para asegurar el mantenimiento de la intensidad de ejercicio adecuada durante el tiempo de trabajo $(\geq 85 \%$ de la frecuencia cardíaca máxima teórica). Los ejercicios realizados fueron Burpee _ ejercicio completo compuesto de una sentadilla, un salto y una flexión _ , skipping _ realización de carrera en el lugar, sin desplazamiento, elevando las rodillas, con gran frecuencia de cambio de piernas _, Jumping Jacks _ ejercicio de coordinación en el que realizando un salto se deben de alternar apertura de piernas sobrepasando ancho de caderas y brazos sobrepasando altura de la cabeza y en el siguiente salto cerrar piernas y brazos _ y Sprint _ señalando en el suelo 2 líneas de 20 metros, separadas entre sí 5 metros, los participantes tendrían que completar el máximo número posible de carrera de una línea a la otra, tocando la cinta del suelo

\section{Condición no C-HIIT}

La condición de no entrenamiento realizada por el Grupo Control consistió en ver 20 minutos del documental Atlantis Rising (National Geographic, 2017). Se escogió este programa para entretener a los jóvenes de forma divertida con un contenido apropiado para su edad, evitando escenas indebidas.

\section{Procedimiento}

Los participantes fueron captados por medio de las Asociaciones de TDAH de la Comunidad de Andalucía. El comunicado le fue enviado vía email a los familiares de los niños diagnosticados inscritos en las asociaciones. Se realizó una reunión informativa en la que se explicó el proyecto. En esa reunión los padres o tutores legales de los niños que estaban interesados en participar cumplimentaron un informe en el que autorizaban la participación de los niños. Se dividieron al azar los niños en dos grupos, Grupo Control y Grupo Experimental. Fueron citados dos días diferentes (dos sábados consecutivos, a las 9h). El Grupo control visualizó durante 20 minutos el video documental Atlantis Rising (National Geographic, 2017). El Grupo Experimental realizó la sesión HIIT. 
Los padres no fueron informados previamente de la condición Control-Experimental, para garantizar que todos participantes aportaran vestimenta y calzado adecuados. Las indicaciones previas al inicio de cada sesión fueron: no comer una hora antes, no hacer actividad física 24 horas antes y no modificar la medicación que siguen con normalidad. Al llegar al laboratorio el primer día, los padres firmaron un consentimiento y también cumplimentaron una hoja sociodemográfica mientras sus hijos realizaban la intervención. Los niños fueron medidos y pesados conforme llegaban al laboratorio. Las pruebas previas y posteriores se realizaron en un gabinete con escritorios individuales. Todas las pruebas fueron hechas con 'bolígrafo y papel' y administradas por grupo. Durante las pruebas, un investigador especializado dio las instrucciones y realizó el seguimiento del tiempo. Mientras, dos asistentes de investigación observaron y dieron solución a posibles dudas y problemas _ del tipo problemas al escribir con el bolígrafo, confusión de línea etc. _. El Grupo Experimental que practicó HIIT lo hizo a una intensidad cercana al $85 \%$ de la frecuencia cardiaca máxima teórica. Este esfuerzo siempre fue bien tolerado por los adolescentes y se controlaba de forma directa proyectando las frecuencias en la pared utilizando un proyector. Cada familia recibió un informe individualizado con los resultados de su hijo.

\section{Análisis estadístico}

Los datos se muestran como medias y desviaciones estándar de los percentiles. Antes del análisis se realizaron pruebas de distribución y homogeneidad (Kolmogorov-Smirnov y Levene), se comprobó que se cumplen la normalidad y la homogeneidad. A continuación, se realizó el test TStudent para muestras apareadas, considerando un intervalo de confianza al 95\%, para valorar los cambios pre-post de la sesión de 16 minutos de entrenamiento HIIT en las variables dependientes percentil del número total de Aciertos, el número de Omisiones y el número de Comisiones. El análisis de varianza de medidas repetidas (ANOVA) 2 veces (pre-test vs. post-test) $\times 2$ grupos (Control vs. Experimental) se usaron para analizar el efecto de 16 minutos de la sesión HIIT. Se usaron como variables dependientes los percentiles correspondientes a los resultados los percentiles de total de Aciertos, número de errores por Omisiones y Comisiones. El grupo se usó como factor fijo. El análisis post-hoc fue ajustado por Bonferroni. El tamaño del efecto se calculó e informó como un valor 2 parcial para las evaluaciones de ANOVA. Estos análisis se llevaron a cabo por separado para cada variable dependiente. Llevamos a cabo todos Ios análisis mediante el uso de IBM-SPSS versión 20.0 para Windows, y el nivel de significación se estableció en $p<.05$.

\section{RESULTADOS}

Análisis T-Student nos muestra las diferencias inter grupos pre-post. Podemos observar en el Grupo Control no hubo cambios significativos ni en total de Aciertos, ni en errores por Comisión ( $p$ $>.05$ ), sin embargo, hubo un descenso significativo de los resultados en número de errores por Omisión $(p=.009)$. En el Grupo Experimental hubo cambios altamente significativos en las tres variables aumentando en número total de Aciertos $(p<.001)$ y disminuyendo el número de errores cometidos por Omisión $(p<.001)$ y Comisión $(p<.001)$.

El análisis de ANOVA mostró un efecto de interacción grupo $x$ tiempo en las principales variables del test d2 $\left(\mathrm{F}(1,18)=26.88, p=.001\right.$, partial $\eta^{2}=0.60 ; \mathrm{F}(1,18)=57.38, p=.001$, partial $\eta^{2}$ $=0.761$ y $\mathrm{F}(1,18)=55.78, p=.001$, partial $\eta^{2}=0.76$ para los percentiles total Aciertos, Omisiones y Comisiones, respectivamente. Un análisis más específico, mostró en el Grupo Experimental incrementó significativamente el nivel de atención, aumentó el número total de Aciertos $(\Delta 4.90 \pm 10.62$ vs. $26.70 \pm 8.00 p<.001$, Figura $2 A)$ y disminuyó el número de errores por número de Omisión $(\Delta$ $-10.30 \pm 9.88$ vs. $18.00 \pm 6.45 p<.001$, Figura $2 \mathrm{~B})$ y Comisión $(\Delta-2.20 \pm 7.49$ vs. $23.60 \pm 7.95 p$ $<.001$, Figura $2 \mathrm{C}$ ) frente al Grupo Control. 
Tabla 2.

Cambios en las variables principales después del programa de intervención. Los valores se expresan como media \pm desviación estándar.

\begin{tabular}{|c|c|c|c|c|c|c|c|c|c|c|c|c|}
\hline \multirow[b]{3}{*}{$\begin{array}{l}\text { D2 Percentil } \\
\text { Total Aciertos }\end{array}$} & \multicolumn{6}{|c|}{$\begin{array}{c}\text { Control } \\
(n=10)\end{array}$} & \multicolumn{6}{|c|}{$\begin{array}{c}\text { Experimental } \\
(n=10)\end{array}$} \\
\hline & \multicolumn{3}{|c|}{ Pre } & \multicolumn{3}{|c|}{ Post } & \multicolumn{3}{|c|}{ Pre } & \multicolumn{3}{|c|}{ Post } \\
\hline & 37.00 & \pm & 16.38 & 41.90 & \pm & 13.88 & 37.90 & \pm & 16.40 & 64.60 & \pm & $20.56^{* * *}$ \\
\hline $\begin{array}{l}\text { D2 Percentil } \\
\text { Omisiones }\end{array}$ & 45.80 & \pm & 9.05 & 35.50 & \pm & $8.32 * *$ & 41.00 & \pm & 9.12 & 59.00 & \pm & $13.81 * * *$ \\
\hline $\begin{array}{l}\text { D2 Percentil } \\
\text { Comisión }\end{array}$ & 34.70 & \pm & 9.00 & 32.50 & \pm & 5.89 & 30.40 & \pm & 10.31 & 54.00 & \pm & $8.76^{* * *}$ \\
\hline
\end{tabular}

Nota: HIIT $=$ High Intensity Interval Training.

${ }^{k} p<.05, * * p<.01, * * * p<.001, \mathrm{~T}$-Student para muestras relacionadas.

Figura 1. Diferencia de medias \pm DS $(95 \%$ IC) en las variables principales tras la intervención. Nivel de significación entre grupos: ${ }^{\star \star \star} p<.001$

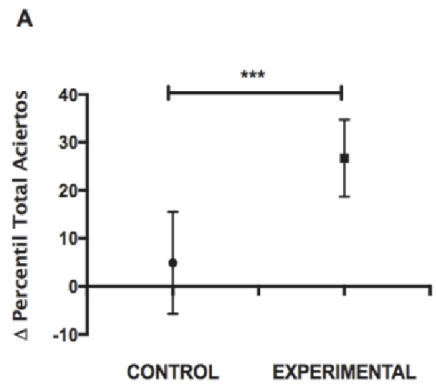

B

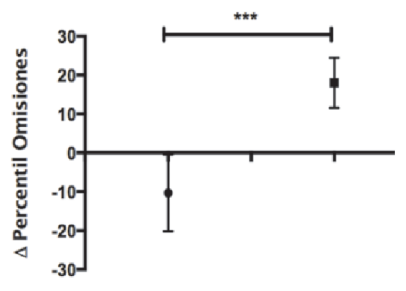

CONTROL
C

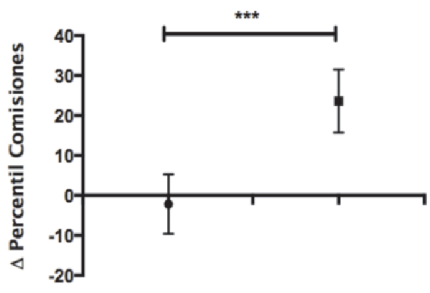

CONTROL EXPERIMENTAL

\section{DISCUSIÓN}

El objetivo de este estudio fue analizar el efecto inmediato de 16 minutos de entrenamiento HIIT en el nivel de atención en niños diagnosticados TDAH. Los resultados obtenidos han mostrado mejoras inmediatas en la atención selectiva, aumentando el número total de respuestas acertadas y disminuyendo el número total de errores cometidos por omisión o comisión. No se observaron efectos negativos en ninguna variable y no se produjeron lesiones durante la sesión de AF. 
Estos resultados son similares a los hallados por Piepmeier et al. (2015), en un estudio con 32 adolescentes _ 18 diagnosticados TDAH y 14 que no presentaban dificultades de aprendizaje _. Tras una intervención de 30 minutos de pedaleo en cicloergometro, a intensidad moderada-vigorosa _ $62-72 \%$ de la frecuencia cardiaca máxima teórica _ , obtuvieron mejoras en la atención selectiva _ Tower of London y Stroop _ , pero no en el conjunto general de funciones ejecutivas. Estos resultados han sido corroborados también por revisiones sistemáticas y metaanálisis publicados durante los últimos años (Grassmann, Alves, Santos-Galduróz y Galduróz, 2014; Suarez-Manzano, Ruiz-Ariza, De la torre-Cruz y Martínez López, 2018) que muestran un efecto positivo de la práctica de AF a intensidad baja o moderada sobre las funciones ejecutivas. Por consiguiente, el principal hallazgo del presente estudio, es que la realización de 16 minutos de un entrenamiento HIIT tiene un efecto inmediato similar al que produce una sesión de AF a intensidad moderada 0 de carácter deportivo de una duración aproximada de 60 minutos, siendo por consiguiente una alternativa más eficiente para que los niños en etapa de Educación Primaria Obligatoria puedan beneficiarse de los efectos del movimiento.

A raíz de estos resultados nos preguntamos si este efecto a lo largo del tiempo se mantiene 0 desaparece. Verret, Guay, Berthiaume, Gardiner y Beliveau (2012) en un estudio de 10 semanas de intervención, encontraron mejoras en atención, además de incremente de inhibición de respuesta y procesamiento de información. La intervención consistió en la realización de tres sesiones semanales de AF de carácter moderado durante 45. También nos preguntamos entonces, ¿cuál sería la frecuencia idónea de las sesiones? ¿a qué se deben estas mejoras en el nivel de atención? Hoy por hoy no podemos dar respuesta a la primera pregunta, pero sí que recientes estudios afirman descubrimientos que explican el porqué de esta mejora. Sabemos que los niveles de dopamina en niños con dificultades de aprendizaje son más bajos y es esta misma evidencia la que se asocia a los bajos niveles de atención (Woleaich et al., 2005). Está demostrado que la práctica de AF aumenta los niveles de dopamina (Gapin, Labban y Etnier, 2011) y reduce el nivel de catecolaminas, Iogrando un menor nivel de ansiedad y estrés (Wigal et al., 2003) lo que permite un mayor autocontrol de emocional. Además, el Ejercicio Físico produce inmediatamente la activación del factor neurotrófico derivado del cerebro, respuesta neuroquímica que desempeña un papel fundamental en niños con síntomas asociados al TDAH, regula la salud celular y es el responsable directo de la diferenciación celular, la plasticidad cerebral y el desarrollo de las funciones ejecutivas (Chen et al., 2017).

\section{FORTALEZAS Y LIMITACIONES DEL ESTUDIO}

Este estudio ha empleado un diseño experimental aleatorizado, creando dos grupos independientes (Grupo Control vs Grupo Experimental), por lo tanto, los resultados son comparables entre grupos. Además, este estudio incluye una muestra más elevada que la presentada por estudios anteriores. No se modificó la medicación de los participantes. El programa empleado _ HIIT _ es un método de entrenamiento que requiere poco tiempo, de fácil de guiar, y no supone elevados costes ya que requiere poco material y espacio reducido. El empleo de monitor de frecuencia cardiaca hace que este sea un método adecuado para niños con dificultades de aprendizaje. La utilización de monitores de frecuencia cardíaca además nos ayuda a controlar el mantenimiento correcto de la intensidad del ejercicio. Sin embargo, por limitaciones de tiempo y posible efecto de aprendizaje del test, no se han podido analizar re-test transcurridas por ejemplo 24 y 48 horas. Además, la escasez de estudios que analicen el efecto de AF a intensidad vigorosa sobre la atención y concretamente, en niños diagnosticados TDAH, ha dificultado la realización de comparaciones directas de los resultados obtenidos en este estudio con los resultados arrojados por otras investigaciones. 


\section{CONCLUSIONES Y FUTURAS LÍNEAS DE INVESTIGACIÓN}

Podemos concluir que la realización de 16 minutos de HIIT tiene un efecto inmediato de mejora en la atención, aumentando el número total de Aciertos y disminuyendo el número total errores por Omisión o Comisión. Esto nos lleva a pensar que, la promoción de la práctica de Ejercicio Físico al menos a intensidad Moderada-Vigorosa ( $\approx 85 \%$ de la frecuencia cardiaca máxima teórica), con una duración de al menos 16 minutos, en los niños con problemas de atención y en especial los jóvenes diagnosticados TDAH, podría ayudar a mejorar los resultados en tareas que requieran mayores niveles de atención. Una buena iniciativa podría ser comentar las materias escolares que requieran altos niveles de atención con este tipo de AF. Sin embargo, más estudios son necesarios para analizar la duración exacta de este efecto inmediato beneficioso en el ámbito escolar, así como identificar las posibles variables que puedan influir, como el tipo de ejercicios, carácter cooperativo, lúdico, cognitivo, temperatura del entorno y empleo de diferentes materiales.

\section{AGRADECIMIENTOS}

El autor quiere mostrar su agradecimiento a los participantes y a sus padres, que han posibilitado su asistencia al estudio. A las Asociaciones de la comunicación de Andalucía por su colaboración activa. A los departamentos de la Universidad de Jaén que han prestado su apoyo y recursos, Departamento de Didáctica de la Expresión Plástica, Musical y Corporal y Departamento de Psicología. La colaboración de todos ellos ha posibilitado la realización de este estudio.

\section{FINANCIACIÓN}

Este documento ha sido parcialmente respaldado por el Programa de Formación de Profesorado Universitario, implementado por el Ministerio de Educación, Cultura y Deporte del Gobierno de España [grant number: AP-2016-07226].

\section{BIBLIOGRAFÍA}

American Psychiatric Association (2013). Diagnostic and Statistical Manual of Mental Disorders (DSM-5®). American Psychiatric Pub

Chen, C., Nakagawa, S., An, Y., Ito, K., Kitaichi, Y., y Kusumi, I. (2017). The exercise-glucocorticoid paradox: How exercise is beneficial to cognition, mood, and the brain while increasing glucocorticoid levels. Frontiers in Neuroendocrinology, 44, 83-102. D0I: 10.1016/j.yfrne.2016.12.001

Cohen J (1998) Statistical Power Analysis for the Behavioral Sciences. Mahwah, NJ: Lawrence Erlbaum Associates.

Costigan, S. A., Eather, N., Plotnikoff, R. C., Hillman, C. H., y Lubans, D. R. (2016). High-Intensity Interval Training for Cognitive and Mental Health in Adolescents. Medicine \& Science in Sports \& Exercise, 48(10), 1985-1993. DOI: 10.1249/MSS.0000000000000993

Egger, H. L., y Angold, A. (2006). Common emotional and behavioral disorders in preschool children: presentation, nosology, and epidemiology. Journal of Child Psychology and Psychiatry, 47(3 4), 313-337. DOI: 10.1111/j.1469-7610.2006.01618.x

Gapin, J. I., Labban, J. D., y Etnier, J. L. (2011). The effects of physical activity on attention deficit hyperactivity disorder symptoms: The evidence. Preventive Medicine, 52, S70-S74. DOI: 10.1016/j.ypmed.2011.01.022

Hamre, B. K., y Pianta, R. C. (2001). Early teacher-child relationships and the trajectory of children's school outcomes through eighth grade. Child development, 72(2), 625-638. DOI: 10.1111/14678624.00301

Karvonen, M. J., Kentala, E., y Mustala, O. (1957). The effects of training on heart rate: A longitudinal study. Annales Medicinae Experimentalis Et Biologiae Fenniae, 35, 307-315. 
Piepmeier, A T, Shih, C. H., Whedon, M., Williams, L. M., Davis, M. E., Henning, D. A., ... Etnier, J. L. (2015). The effect of acute exercise on cognitive performance in children with and without ADHD. Journal of Sport and Health Science, 4(1), 97-104. D0I: 10.1016/j.jshs.2014.11.004

Polanczyk, G., de Lima, M. S., Horta, B. L., Biederman, J., y Rohde, L. A. (2007). The worldwide prevalence of ADHD: a systematic review and metaregression analysis. The American journal of psychiatry, 164(6), 942-948. D0I:10.1176/ajp.2007.164.6.942

Pontifex, M. B., Saliba, B. J., Raine, L. B., Picchietti, D. L., y Hillman, C. H. (2013). Exercise improves behavioral, neurocognitive, and scholastic performance in children with attention-deficit/hyperactivity disorder. The Journal of pediatrics, 162(3), 543-551. DOI:10.1016/j. jpeds.2012.08.036

Seisdedos, N. (2012). Adaptación española D2, test de atención de Brickenkamp (4ª Edición revisada). Madrid: TEA Editociones.

Suarez-Manzano, S.; Ruiz-Ariza, A.; De la Torre-Cruz, M.J.; Martínez López, E.J. (2018) Acute and chronic effect of physical activity on cognition and behaviour in young people with ADHD: A systematic review of intervention studies, Research in Developmental Disabilities, 77, 12-23. D0I: 10.1016/j.ridd.2018.03.015

Vanhelst, J., Béghin, L., Duhamel, A., Manios, Y., Molnar, D., De Henauw, S., ... y Gottrand, F. (2016). Physical Activity Is Associated with Attention Capacity in Adolescents. The Journal of Pediatrics, 168, 126-131. DOI: 10.1016/j.jpeds.2015.09.029

Wigal, S. B., Nemet, D., Swanson, J. M., Regino, R., Trampush, J., Ziegler, M. G., y Cooper, D. M. (2003). Catecholamine response to exercise in children with attention deficit hyperactivity disorder. Pediatric research, 53(5), 756-761. DOI: 10.1203/01.PDR.0000061750.71168.23

Wittchen, H.U., Jacobi, F., Rehm, J., Gustavsson, A., Svensson, M., Jönsson, B., ... y Faravelli, C. (2011). The size and burden of mental disorders and other disorders of the brain in Europe 2010. European Neuropsychopharmacology, 21(9), 655-679. D0I: 10.1016/j.euroneuro.2011.07.018

Wolraich, M. L., Wibbelsman, C. J., Brown, T. E., Evans, S. W., Gotlieb, E. M., Knight, J. R., ... y Wilens, T. (2005). Attention-deficit/hyperactivity disorder among adolescents: a review of the diagnosis, treatment, and clinical implications. Pediatrics, 115(6), 1734-1746. DOI: 10.1542/peds.2004-1959 